\title{
EVALUATION OF CERTAIN INTEGRALS IN TERMS OF GENERALIZED KAMPE DE FERIET FUNCTION AND LAURICELLA FUNCTION $F_{D}^{(n)}$
}

\author{
MEGUMI SAIGO AND R. K. RAINA .
}

\begin{abstract}
A.bstract. The paper is concerned with the evaluation of three integrals involving general polynomial systems and a rational function of trigonometric functions. Our new formulas reduce to many known formulas.
\end{abstract}

\section{Introduction}

A general polynomial system $S_{n}^{m}(x)$ was introduced by Srivastava [7], and is defined as

$$
S_{n}^{m}(x)=\sum_{j=0}^{[n / m]}(-n)_{m j} A(n, j) \frac{x^{j}}{j !}
$$

where $m$ is a positive integer, $n$ a non-negative integer, $A(n, i)$ an arbitrary sequence of real or complex numbers. The symbol $(\alpha)_{n}$ stands for the factorial function and is defined by

$$
(\alpha)_{n}=\frac{\Gamma(\alpha+n)}{\Gamma(\alpha)}= \begin{cases}\alpha(\alpha+1) \cdots(\alpha+n-1), & n=1,2, \cdots \\ 1, & n=0\end{cases}
$$

The polynomial system reduces to several classes of orthogonal polynomials, and details may be found in [7].

The generalized Kampé de Fériet function (see [8, p.65]) is defined by

$$
F_{q: q_{1} ; \cdots ; q_{n}}^{p: p_{1} ; \cdots ; p_{n}}\left[\begin{array}{c}
\left(\alpha_{p}\right):\left(\alpha_{p_{1}}^{\prime}\right) ; \cdots ;\left(\alpha_{p_{n}}^{(n)}\right) ; \\
\left(\beta_{q}\right):\left(\beta_{q_{1}}^{\prime}\right) ; \cdots ;\left(\beta_{q_{n}}^{(n)}\right) ;
\end{array} z_{1}, \cdots, z_{n}\right]=\sum_{j_{1}, \cdots, j_{n}=0}^{\infty} \Lambda\left(j_{1}, \cdots, j_{n}\right) \frac{z_{1}^{j_{1}}}{j_{1} !} \cdots \frac{z_{n}^{j_{n}}}{j_{n} !}
$$

Received September 3, 1993.

1991 Mathematics Subject Classification.

Key words and phrases 
where

$$
\Lambda\left(j_{1}, \cdots, j_{n}\right)=\frac{\prod_{i=1}^{p}\left(\alpha_{i}\right)_{j_{1}+\cdots+j_{n}} \prod_{i=1}^{p_{1}}\left(\alpha_{i}^{\prime}\right)_{j_{1}} \cdots \prod_{i=1}^{p_{n}}\left(\alpha_{i}^{(n)}\right)_{j_{n}}}{\prod_{i=1}^{q}\left(\beta_{i}\right)_{j_{1}+\cdots+j_{n}} \prod_{i=1}^{q_{1}}\left(\beta_{i}^{\prime}\right)_{j_{1}} \cdots \prod_{i=1}^{q_{n}}\left(\beta_{i}^{(n)}\right)_{j_{n}}}
$$

For convergence of the series (1.3), it is assumed that $q+q_{i}-p-p_{i} \geqq 0(i=1, \cdots, n)$. When the equality holds in this condition, the series (1.3) converges if either $p>q$ and $\left|z_{1}\right|^{1 /(p-q)}+\cdots+\left|z_{n}\right|^{1 /(p-q)}<1$ or $p \leqq q$ and $\max \left\{\left|z_{1}\right|, \cdots,\left|z_{n}\right|\right\}<1$.

The present paper is intended to evaluate three integrals. The first one involving a product of polynomials (1.1) in the integrand is seen to yield the value in terms of generalized Kampé de Fériet function (1.3) after specialization of the arbitrary sequence. The second integral evaluates a family of integrals involving the product of an algebraic function and the multidimensional analogue of (1.1). The last one is an integral of a certain rational function of trigonometric functions and is represented by the Lauricella function $F_{D}^{(n)}$, where $F_{D}^{(n)}$ is known to be a special case of the Kampé de Fériet function:

$$
F_{D}^{(n)}\left[\begin{array}{c}
\alpha, \beta_{1}, \cdots, \beta_{n} ; \\
\gamma_{1}, \cdots, \gamma_{n} ;
\end{array} z_{1}, \cdots, z_{n}\right]=F_{0: 1 ; \cdots ; 1}^{1: 1 ; \cdots ; 1}\left[\begin{array}{r}
\alpha, \beta_{1}, \cdots, \beta_{n} ; \\
\gamma_{1}, \cdots, \gamma_{n} ;
\end{array}\right]
$$

The formulas given in this paper are useful and can be used in applied branches of physics and engineering. We, however, deduce only few interesting integrals from our integral formulas.

\section{First Integral}

We derive the following integral:

$$
\begin{aligned}
& \int_{p}^{q} \frac{(x-p)^{\lambda-1}(q-x)^{\mu-1}}{[q-p+a(x-p)+b(q-x)]^{\lambda+\mu}} S_{n_{1}}^{m_{1}}\left(\frac{p-x}{q-x}\right) \cdots S_{n_{r}}^{m_{r}}\left(\frac{p-x}{q-x}\right) d x \\
= & \frac{1}{(q-p)(1+a)^{\lambda}(1+b)^{\mu}} B(\lambda, \mu) \\
& \cdot \sum_{j_{1}=0}^{\left[n_{1} / m_{1}\right]} \cdots \sum_{j_{r}=0}^{\left[n_{r} / m_{r}\right]} \prod_{i=1}^{r}\left\{\frac{\left(-n_{i}\right)_{m_{i} j_{i}}}{j_{i} !} A_{i}\left(n_{i}, j_{i}\right)\right\}\left(\frac{1+b}{1+a}\right)^{\sum j_{i}} \frac{(\lambda) \sum j_{i}}{(1-\mu) \sum j_{i}},
\end{aligned}
$$

provided that $\operatorname{Re}(\lambda)>0, \operatorname{Re}(\mu)>0, a$ and $b$ are complex numbers with $1+a \neq 0,1+b \neq$ 0 , and $x \in[p, q]$ such that $q-p+a(x-p)+b(q-x) \neq 0$, where $\sum j_{i}$ means the sum of non-negative integers $j_{1}+\cdots+j_{r}, S_{n}^{m}(x)$ is the general polynomial system defined in (1.1) with coefficients $A(n, j)$, and $B(\lambda, \mu)$ is the Beta function.

Proof. Using (1.1) and expanding each polynomial, we have 


$$
\begin{aligned}
\text { L.H.S. }= & \sum_{j_{1}=0}^{\left[n_{1} / m_{1}\right]} \cdots \sum_{j_{r}=0}^{\left[n_{r} / m_{r}\right]} \prod_{i=1}^{r}\left\{\frac{\left(-n_{i}\right)_{m_{i} j_{i}}}{j_{i} !} A_{i}\left(n_{i}, j_{i}\right)\right\} \\
& \cdot(-1)^{\sum j_{i}} \int_{p}^{q} \frac{(x-p)^{\lambda+\sum j_{i}-1}(q-x)^{\mu-\sum j_{i}-1}}{[q-p+a(x-p)+b(q-x)]^{\lambda+\mu}} d x .
\end{aligned}
$$

By appealing to the following variation of the formula in $[5$, p.70, Eqn.(3.1.3)]:

$$
\int_{p}^{q} \frac{(t-p)^{\lambda-1}(q-t)^{\mu-1}}{[q-p+a(t-p)+b(q-t)]^{\lambda+\mu}} d t=(q-p)^{-1}(1+a)^{-\lambda}(1+b)^{-\mu} B(\lambda, \mu)
$$

and invoking the simple relation

$$
\Gamma(\alpha-n)=\frac{(-1)^{n} \Gamma(\alpha)}{(1-\alpha)_{n}}, \alpha=0, \pm 1, \pm 2, \cdots,
$$

we arrive at the desired right hand member of (2.1).

Deduction. On selecting the arbitrary sequences

$$
A_{i}\left(n_{i}, j_{i}\right)=\frac{\prod_{s=1}^{p_{i}}\left(a_{s}^{(i)}\right)_{j_{i}}}{\prod_{s=1}^{q_{i}}\left(b_{s}^{(i)}\right)_{j_{i}}}
$$

for $i=1, \cdots, r$, and noting each polynomial

$$
S_{n_{i}}^{m_{i}}(x)=B_{n_{i}}^{m_{i}}\left(x m_{i}^{m_{i}}\right)=\sum_{j_{i}=0}^{\left[n_{i} / m_{i}\right]}\left(-n_{i}\right)_{m_{i} j_{i}} \frac{\prod_{s=1}^{p_{i}}\left(a_{s}^{(i)}\right)_{j_{i}}}{\prod_{s=1}^{q_{i}}\left(b_{s}^{(i)}\right)_{j_{i}}} \frac{x^{j_{i}}}{j_{i} !} \quad(i=1, \cdots, r),
$$

being the Brafman polynomial [1, p.186], (2.1) gives the integral

$$
\begin{aligned}
& \int_{p}^{q} \frac{(x-p)^{\lambda-1}(q-x)^{\mu-1}}{[q-p+a(x-p)+b(q-x)]^{\lambda+\mu}} B_{n_{1}}^{m_{1}}\left(m_{1}^{m_{1}} \frac{p-x}{q-x}\right) \cdots B_{n_{r}}^{m_{r}}\left(m_{r}^{m_{r}} \frac{p-x}{q-x}\right) d x \\
= & \frac{1}{(q-p)(1+a)^{\lambda}(1+b)^{\mu}} B(\lambda, \mu) \\
& \cdot F_{1: q_{1}+m_{1} ; \cdots ; q_{r}+m_{r}}^{1: p_{1}+m_{1} ; \cdots ; p_{r}+m_{r}}\left[\begin{array}{ccc}
\lambda: \Delta\left(m_{1} ;-n_{1}\right),\left(a_{p_{1}}^{\prime}\right) ; \cdots ; & \Delta\left(m_{r} ;-n_{r}\right),\left(a_{p_{r}}^{(r)}\right) ; \\
1-\mu: & \left(b_{q_{1}}^{\prime}\right) ; \cdots ; & \left(b_{q_{r}}^{(r)}\right) ; \\
& \left.m_{1}^{m_{1}}\left(\frac{1+b}{1+a}\right), \cdots, m_{r}^{m_{r}}\left(\frac{1+b}{1+a}\right)\right],
\end{array}\right.
\end{aligned}
$$

valid under the conditions stated with $(2.1)$, where $\Delta(m ; n)$ means the array $\frac{n}{m}, \frac{n+1}{m}, \cdots$, $\frac{n+m-1}{m}$, and we have noted that

$$
(n)_{m_{j}}=m^{m_{j}}\left(\frac{n}{m}\right)_{j}\left(\frac{n+1}{m}\right)_{j} \ldots\left(\frac{n+m-1}{m}\right)_{j} .
$$


In view of the definition $[8, \mathrm{p} .91$, Eqn. (16)(iii)] we have when $p=-1, q=1$ that

$$
B_{n}^{1}\left(\frac{x+1}{x-1}\right)={ }_{2} F_{1}\left[\begin{array}{rr}
-n,-\alpha-n ; & \\
\beta+1 ; & \frac{x+1}{x-1}
\end{array}\right]=\left(\begin{array}{c}
\beta+n \\
n
\end{array}\right)^{-1}\left(\frac{2}{x-1}\right)^{n} P_{n}^{(\alpha, \beta)}(x),
$$

where ${ }_{2} F_{1}$ is the Gauss function and $P_{n}^{(\alpha, \beta)}$ the Jacobi polynomial. With this specialization we get from (2.5) the following integral involving the product of Jacobi polynomials:

$$
\begin{aligned}
& \int_{-1}^{1} \frac{(1+x)^{\lambda-1}(1-x)^{\mu-\sum n_{i}-1}}{[2+a(1+x)+b(1-x)]^{\lambda+\mu}} P_{n_{1}}^{\left(\alpha_{1}, \beta_{1}\right)}(x) \cdots P_{n_{r}}^{\left(\alpha_{r}, \beta_{r}\right)}(x) d x \\
= & \frac{1}{2(1+a)^{\lambda}(1+b)^{\mu}} B(\lambda, \mu)\left(-\frac{1}{2}\right)^{\sum n_{i}} \prod_{j=1}^{r}\left(\begin{array}{c}
\beta_{j}+n_{j} \\
n_{j}
\end{array}\right) \\
& \cdot F_{1: 1 ; \cdots ; 1}^{1: 2 ; \cdots ; 2}\left[\begin{array}{rrr}
\lambda: & -n_{1},-n_{1}-\alpha_{1} ; \cdots ; & -n_{r},-n_{r}-\alpha_{r} ; \\
1-\mu: & \beta_{1}+1 ; \cdots ; & \frac{1+b}{1+a}, \cdots, \frac{1+b}{1+a}
\end{array}\right] .
\end{aligned}
$$

If $\alpha=\beta=0$, then $P_{n}^{(0,0)}(x)=P_{n}(x)$ is the Legendre polynomial and we have from (2.7) when $\lambda=1, \mu=1+\sum n_{i}, a=b=0$ the following known formula [4, p.1456, Eqn.(4)] after little simplification:

$$
\int_{-1}^{1} P_{n_{1}}(x) \cdots P_{n_{r}}(x) d x=\frac{2}{1+\sum n_{i}} \sum_{j_{1}=0}^{n_{1}} \cdots \sum_{j_{r}=0}^{n_{r}}(-1) \sum j_{i} \frac{\left(\begin{array}{c}
n_{1} \\
j_{1}
\end{array}\right)^{2} \cdots\left(\begin{array}{c}
n_{r} \\
j_{r}
\end{array}\right)^{2}}{\left(\begin{array}{c}
\sum n_{i} \\
\sum j_{i}
\end{array}\right)} .
$$

\section{Second Integral}

Define a polynomial set by

$$
H_{n}^{s_{1}, \cdots, s_{r}}\left(x_{1}, \cdots, x_{r}\right)=\sum_{j_{1}, \cdots, j_{r}=0}^{J \leqq n}(-n)_{J} C\left(n ; j_{1}, \cdots, j_{r}\right) \prod_{i=1}^{r} \frac{x_{i}^{j_{i}}}{j_{i} !}
$$

where $s_{i}(i=1, \cdots, r)$ are positive integers,

$$
J=\sum_{i=1}^{r} s_{i} j_{i}
$$

and the coefficients $C\left(n ; j_{i}, \cdots, j_{r}\right)$ are multiple sequences for nonnegative integers $j_{i}(i=$ $1, \cdots, r)$ of arbitrary constants. 
We establish the following integral:

$$
\begin{aligned}
& \int_{0}^{b} x^{\lambda-1}\left(x^{m}+c\right)^{-\alpha} H_{n}^{s_{1}, \cdots, s_{r}}\left(-\frac{a_{1}^{m}}{x^{m}+c}, \cdots,-\frac{a_{r}^{m}}{x^{m}+c}\right) d x \\
= & \frac{b^{\lambda}}{\lambda c^{\alpha}} \sum_{j_{1}, \cdots, j_{r}=0}^{J \leqq n}(-n)_{J} \frac{C\left(n ; j_{1}, \cdots, j_{r}\right)}{j_{1} ! \cdots j_{r} !}(-c)^{-\sum j_{i}} a_{1}^{m j_{1}} \cdots a_{r}^{m j_{r}} \\
& \cdot \sum_{k=0}^{\infty} \frac{(\alpha) \sum j_{i}+k}{(\alpha) \sum j_{i}} \frac{\left(\frac{\lambda}{m}\right)_{k}}{k !\left(\frac{\lambda}{m}+1\right)_{k}}\left(-\frac{b^{m}}{c}\right)^{k},
\end{aligned}
$$

provided that $\min \left\{a_{1}, \cdots, a_{r}, b, c\right\}>0, m$ is a positive integer, $\alpha$ is a complex number and $\operatorname{Re}(\lambda)>0$.

Proof. Invoking the definition (3.1), we have on integrating term by term

$$
\begin{aligned}
\text { L.H.S. of }(3.3)= & \sum_{j_{1}, \cdots, j_{r}=0}^{J \leqq n}(-n)_{J} \frac{C\left(n ; j_{1}, \cdots, j_{r}\right)}{j_{1} ! \cdots j_{r} !}(-1)^{-\sum j_{i}} a_{1}^{m j_{1}} \cdots a_{r}^{m j_{r}} \\
& \cdot \int_{0}^{b} x^{\lambda-1}\left(x^{m}+c\right)^{-\alpha-\sum j_{i}} d x .
\end{aligned}
$$

The simple integral on the right side can easily be evaluated in terms of the Gauss function ${ }_{2} F_{1}$, and the resulting expression involving the $(r+1)$ series is the desired R.H.S. of (3.3).

Deduction. If we set

$$
C\left(n ; j_{1}, \cdots, j_{r}\right)=\Lambda\left(j_{1}, \cdots, j_{r}\right)
$$

for $\Lambda$ being defined in (1.4) with $p=q=0$, then we get when $s_{i}=1(i=1, \cdots, r)$ in $(3.3)$ :

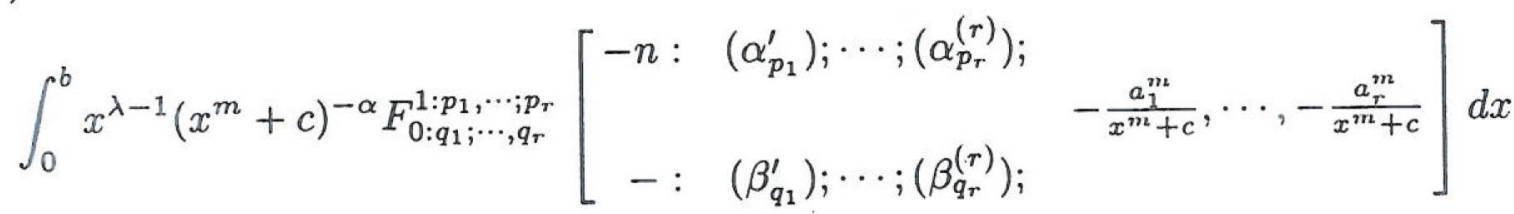

$$
\begin{aligned}
& =\frac{b^{\lambda}}{\lambda c^{\alpha}} \sum_{j_{1}, \cdots, j_{r}=0}^{\sum j_{i} \leqq n}(-n) \sum j_{i} \frac{\prod_{s=1}^{p_{1}}\left(\alpha_{s}^{\prime}\right)_{j_{1}} \cdots \prod_{s=1}^{p_{r}}\left(\alpha_{s}^{(r)}\right)_{j_{r}}}{\prod_{s=1}^{q_{1}}\left(\beta_{s}^{\prime}\right)_{j_{1}} \cdots \prod_{s=1}^{p_{r}}\left(\beta_{s}^{(r)}\right)_{j_{r}}} \\
& \cdot(-c)^{\sum j_{i}} \frac{a_{1}^{m j_{1}} \cdots a_{r}^{m j_{r}}}{j_{1} ! \cdots j_{r} !}{ }_{2} F_{1}\left(\alpha+\sum j_{i}, \frac{\lambda}{m} ; 1+\frac{\lambda}{m} ;-\frac{b^{m}}{c}\right) \text {. }
\end{aligned}
$$

Another second integral

$$
\int_{0}^{b} x^{\lambda-1}\left(x^{m}+c\right)^{-\alpha} F_{0: q_{1} ; \cdots, q_{r}}^{1: p_{1}, \cdots ; p_{r}}\left[\begin{array}{cc}
\alpha:\left(\alpha_{p_{1}}^{\prime}\right) ; \cdots ;\left(\alpha_{p_{r}}^{(r)}\right) ; & -\frac{a_{1}^{m}}{x^{m}+c}, \cdots,-\frac{a_{r}^{m}}{x^{m}+c} \\
-:\left(\beta_{q_{1}}^{\prime}\right) ; \cdots ;\left(\beta_{q_{r}}^{(r)}\right) ;
\end{array}\right] d x
$$




$$
=\frac{b^{\lambda}}{\lambda c^{\alpha}} F_{0: q_{1} ; \cdots, q_{r} ; 1}^{1: p_{1}, \cdots ; p_{r} ; 1}\left[\begin{array}{cr}
\alpha:\left(\alpha_{p_{1}}^{\prime}\right) ; \cdots ;\left(\alpha_{p_{r}}^{(r)}\right) ; & \lambda / m ; \\
-:\left(\beta_{q_{1}}^{\prime}\right) ; \cdots ;\left(\beta_{q_{r}}^{(r)}\right) ; & 1+\lambda / m ;
\end{array}\right]
$$

provided that $\min \left\{a_{1}, \cdots, a_{r}, b, c\right\}>0, m$ is a positive integer, and $\operatorname{Re}(\lambda)>0$, and the convergence conditions appropriate to (1.3) for the generalized Kampé de Fériet function are satisfied. For $r=1$, the result reduces to the one given by Saigo and Srivastava [6, p.2, Eqn. (2.1)].

\section{Third integral}

Now we establish:

$$
\begin{aligned}
& \int_{0}^{\varphi} \prod_{i=1}^{n}\left(\frac{\sin ^{2 \sigma_{i}} \theta}{\left(1+k_{i} \sin ^{2} \theta\right)^{h_{i}}}\right) \frac{\sin ^{2 \sigma} \theta}{\left(1-k^{2} \sin ^{2} \theta\right)^{h+1 / 2}} d \theta \\
& =\frac{(\sin \varphi)^{2\left(\sigma+\sum \sigma_{i}\right)+1}}{2\left(\sigma+\sum \sigma_{i}\right)+1} \cdot F_{D}^{(n+2)}\left[\begin{array}{l}
\sigma+\sum \sigma_{i}+\frac{1}{2}, h+\frac{1}{2}, \frac{1}{2}, h_{1}, \cdots, h_{n} ; \\
\sigma+\sum \sigma_{i}+\frac{3}{2}
\end{array}\right. \\
& \left.k^{2} \sin ^{2} \varphi, \sin ^{2} \varphi,-k_{1} \sin ^{2} \varphi, \cdots,-k_{n} \sin ^{2} \varphi\right] \text {, }
\end{aligned}
$$

provided that

$$
\operatorname{Re}\left(\sigma+\sum \sigma_{i}\right)>-\frac{1}{2}, 0 \leqq \varphi \leqq \frac{\pi}{2}, k^{2} \sin ^{2} \varphi<1,\left|k_{i}\right| \sin ^{2} \varphi<1(i=1, \cdots, n) .
$$

Proof. If we set $u=\sin ^{2} \theta / \sin ^{2} \varphi$, then L.H.S. of (4.1) gives

\section{L.H.S.}

$$
\begin{aligned}
= & \frac{1}{2}(\sin \varphi)^{2\left(\sigma+\sum \sigma_{i}\right)+1} \int_{0}^{1} u^{\sigma+\sum \sigma_{i}-1 / 2} \prod_{i=1}^{n}\left(1+u k_{i} \sin ^{2} \varphi\right)^{-h i} \\
& \cdot\left(1-u k^{2} \sin ^{2} \varphi\right)^{-h-1 / 2}\left(1-u \sin ^{2} \varphi\right)^{-1 / 2} d u \\
= & \frac{1}{2}(\sin \varphi)^{2\left(\sigma+\sum \sigma_{i}\right)+1} \sum_{l, m=0}^{\infty} \sum_{m_{1}, \cdots, m_{n}=0}^{\infty} \frac{\left(h+\frac{1}{2}\right) l\left(\frac{1}{2}\right) m}{l ! m !}\left(k^{2} \sin ^{2} \varphi\right)^{l}\left(\sin ^{2} \varphi\right)^{m} \\
& \cdot \frac{\left(h_{1}\right)_{m_{1}} \cdots\left(h_{n}\right)_{m_{n}}}{m_{1} ! \cdots m_{n} !}\left(-k_{1} \sin ^{2} \varphi\right)^{m_{1}} \cdots\left(-k_{n} \sin ^{2} \varphi\right)^{m_{n}} \int_{0}^{1} u^{\sigma+\sum \sigma_{i}-1 / 2+l+m+\sum m_{i}} d u
\end{aligned}
$$

Upon simplification, the R.H.S. on interpreting the series in terms of the fourth Lauricella function involving several variables (1.5), the desired integral (3.1) is arrived at. 
Deduction. We note the following two special cases of the third integral

(i) For $\sigma_{i}=0(i=1, \cdots, n), \sigma=0, h=0$ and $h_{i}=1(i=1, \cdots, n),(4.1)$ reduces to the result given in [3, p.264, Eqn. (8.4.3)].

(ii) Again, if we put $\sigma_{i}=\sigma=k_{i}=0(i=1, \cdots, n)$, then (4.1) gives

$$
\begin{aligned}
\int_{0}^{\varphi} \frac{d \theta}{\left(1-k^{2} \sin ^{2} \theta\right)^{h+1 / 2}} & =\sin \varphi F_{1}\left[\frac{1}{2}, h+\frac{1}{2}, \frac{1}{2} ; \frac{3}{2} ; k^{2} \sin ^{2} \varphi, \sin ^{2} \varphi\right] \\
& =\sin \varphi \sum_{r=0}^{\infty} \frac{\left(h+\frac{1}{2}\right)_{r}\left(\frac{1}{2}\right)_{r}}{\left(\frac{3}{2}\right)_{r} r !}\left(k^{2} \sin ^{2} \varphi\right)^{r}{ }_{2} F_{1}\left[\begin{array}{cc}
\frac{1}{2}+r, \frac{1}{2} ; & \left.\sin ^{2} \varphi\right] \\
\frac{3}{2}+r ; &
\end{array}\right]
\end{aligned}
$$

on using [8, p.53, Eqn.(4)], where $F_{1}$ denotes the first Appell function of two variables. By setting $k^{2}=\lambda^{2} /\left(\lambda^{2}-1\right)$ with $|\lambda|<1$, the integral on the L.H.S. of (4.2) takes the form

$$
\left(1-\lambda^{2}\right)^{h+1 / 2} \int_{0}^{\varphi} \frac{d \theta}{\left(1-\lambda^{2} \cos ^{2} \theta\right)^{h+1 / 2}},
$$

and on further putting $\varphi=\pi / 2$, this integral becomes

$$
\left(1-\lambda^{2}\right)^{h+1 / 2} \int_{0}^{\pi / 2} \frac{d \theta}{\left(1-\lambda^{2} \cos ^{2} \theta\right)^{h+1 / 2}},
$$

and its value thus computed from the R.H.S. of (4.2) yields the known result [2, p.1].

\section{References}

[1] F. Brafman, "Some generating functions of Laguerre and Hermite polynomials," Canad. J. Math. 9(1957), 180-187.

[2] L. F. Epstein, and J. H. Hubell, "Evaluation of a generalized elliptic-type integral," J. Res., 67B (1963), 1.

[3] H. Exton, Multiple Hypergeometric Functions and Applications, Halsted Press (Ellis Horwood, Chichester), John Wiley and Sons, New York-London-Sydney-Toronto, 1978.

[4] J. Gillis, J. Jedwab, and D. Zeilberger, "A combinatorial interpretation of the integral of the product of Legendre polynomials," SIAM J. Math. Anal., 19(1988), 1455-1461.

[5] A. M. Mathai and R. K. Saxena, "Generalized Hypergeometric Functions with Applications in Statistics and Physical Sciences," Lecture Notes in Math., 348, Springer-Verlag, Berlin-HeidelbergNew York, 1973.

[6] M. Saigo and R. Srivastava, "Some new results for radiation-field problems," Fukuoka Univ. Sci. Rep. 20 (1990), 1-13.

[7] H. M. Srivastava, "The Weyl fractional integral of a general class of polynomials," Boll. Un. Mat. Ital. (6), 2B (1983), 219-288.

[8] H. M. Srivastava and H. L. Manocha, A Treatise on Generating Functions, Halsted Press (Ellis Horwood, Chichester), John Wiley and Sons, New York-Chichester-Brisbane-Toronto, 1984.

Department of Applied Mathematics, Faculty of Science Fukuoka University, Fukuoka 814-01, Japan. Department of Mathematics, College of Technology and Agricultural Engineering Rajasthan Agricultrual University, Udaipur 313001, Rajasthan, India. 